\title{
Self-consistent Bogoliubov de Gennes theory of the vortex lattice state in a two-dimensional strong type-II superconductor at high magnetic fields
}

\author{
Vladimir Zhuravlev \\ Schulich Faculty of Chemistry, Technion-Israel Institute of Technology, Haifa 32000, Israel \\ Wenye Duan \\ School of Physics, Peking University, Beijing 100871, China \\ Tsofar Maniv \\ Schulich Faculty of Chemistry, Technion-Israel Institute of Technology, Haifa 32000, Israe*
}

(Dated: November 18, 2016)

\begin{abstract}
A self-consistent Bogoliubov deGennes theory of the vortex lattice state in a $2 \mathrm{D}$ strong type-II superconductor at high magnetic fields reveals a novel quantum mixed state around the semiclassical $\mathrm{H}_{c 2}$, characterized by a well-defined Landau-Bloch band structure in the quasi-particle spectrum and suppressed order-parameter amplitude, which sharply crossover into the well-known semiclassical (Helfand-Werthamer) results upon decresing magnetic field. Application to the 2D superconducting state observed recently on the surface of the topological insulator $\mathrm{Sb}_{2} \mathrm{Te}_{3}$, accounts well for the experimental data, revealing a strong type-II superconductor, with unusualy low carrier density and very small cyclotron mass, which can be realized only in the strong coupling superconductor limit.
\end{abstract}

PACS numbers: 74.78.-w, 74.25.Ha, 74.20.-z, 74.25.Uv

The recent discoveries of surface and interface superconductivity with exceptionally high superconducting (SC) transition temperatures in several material structures [1, 2], 3] promise to stimulate fundamental studies of the phenomenon of strong type-II superconductivity in two-dimensional (2D) and quasi 2D electron systems, particularly under high magnetic fields. 4. In a pure strong type-II superconductor under a uniform magnetic field the quasi particle spectrum is gapless in a broad field range below the upper critical field $H_{c 2}$ [5], [6], 4, where scattering of quasi particles by the vortex lattice interferes with the Landau quantization of the electron motion perpendicular to the magnetic field to form magnetic (Landau) Bloch's bands. In pure 2D, or quasi 2D, strong type-II SC systems, such as that realized in the multilayer system of the organic charge transfer salt $\kappa-(E T)_{2} C u(S C N)_{2}$ [7], under a magnetic field perpendicular to the easy conducting plane, the underlying normal electron spectrum is fully quantized and the effect of the vortex lattice is very pronounced. $2 \mathrm{D}$ vortex lattices can realize in such strongly layered electronic systems due to the presence of weak crystalline disorder [8], [9, where pinning of a few flux lines provide support for the entire vortex lattice against melting under an increasing magnetic field up to the irreversibility line [10. Of special interest in the present paper is the unique situation of the $2 \mathrm{D}$ superconductivity realized in surface states of topological insulators, e.g. $\mathrm{Sb}_{2} \mathrm{Te}_{3}$ [11, where the chemical potential $\mu$ is close to a Dirac point [12] (with Fermi velocity $v$ ) and the cyclotron effective mass, $m^{*}=\mu / v^{2}\left[13\right.$ is a small fraction (e.g. 0.065 in $\mathrm{Sb}_{2} \mathrm{Te}_{3}$ ) of the free electron mass $m_{e}$, resulting in a dramatic enhancement of the cyclotron frequency, $\omega_{c}=e H / m^{*} c$, and the corresponding Landau level (LL) energy spacing.

Due to the suppressed energy dispersion along the magnetic field direction, characterizing the 2D electron system, and the particle-hole symmetry inherent to the SC state, the quasi particle spectrum exhibits peculiar features that are missing in the 3D case. For example, at discrete magnetic field values where the chemical potential is located in the middle of a Landau band, so that the underlying normal state spectrum satisfies particle-hole symmetry, the calculated quasi-particle density of states shows a linear, Dirac-like energy dependence, which reflects topological singularities at the vortex lattice cores 4,,14. Both the enhanced Landau quantization effect and the lucid reflection of the topological singularity at the vortex-lattice cores in the quasiparticle spectrum, 15, [16], point to the great importance of self consistency in the theoretical description of $2 \mathrm{D}$ superconductors at high magnetic fields. The presence of well defined Landau bands in the quasi-particle spectrum, which is reflected as magneto-quantum (MQ) oscillations in the SC order parameter, [17] via the self consistency equation, is expected to significantly alter the semiclassical picture of the SC phase transition at high magnetic fields. 18 In this paper we present, for the first time, results of systematic self-consistent solutions of the Bogoliubove de Gennes (BdG) equations, which addresses these aspects of $2 \mathrm{D}$ superconductivity at high magnetic fields. It is, indeed, found that, due to selfconsistency, the SC pair-potential can be strongly distorted near the vortex-lattice cores, and that the traditional semiclassical picture of a single critical point can be dramatically smeared into an intermediate state of multicritical transition. The self-consistency formalism 

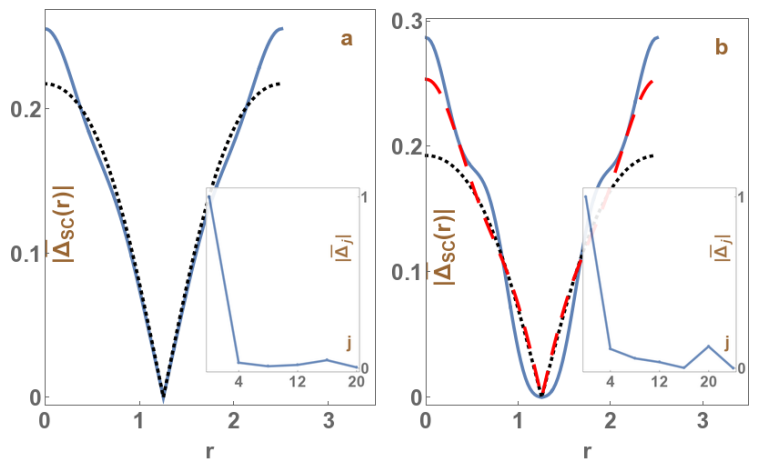

FIG. 1: Color online: (a) Self-consistent $\left|\bar{\Delta}_{S C}(\mathbf{r})\right|$ (i.e. measured in units of $\left.\hbar \omega_{c}\right)$, as a function of coordinate along the diagonal of the unit cell in the (square) vortex lattice (blue solid line), for $n_{F}=9$. The other parameters used in the calculation are: $\lambda=0.75, \hbar \omega_{D} / \mu=0.2$, and $k_{B} T / \mu=10^{-4}$. For comparison, the resulting $\left|\bar{\Delta}_{S C}(\mathbf{r})\right|$ calculated only with $\bar{\Delta}_{j=0}$, which corresponds to the Abrikosov solution, is also shown (dotted line). The inset shows all the nonzero coefficients $\left|\bar{\Delta}_{j}\right|, j=0,4,8,12,16 \leq 2 n_{F}$. (b) Similar to (a) but for $n_{F}=10$. The inset shows the nonzero coefficients $\left|\bar{\Delta}_{j}\right|$ at $j=0,4,8,12,16,20 \leq 2 n_{F}$. Note the parabolic distortion of $\left|\bar{\Delta}_{S C}(\mathbf{r})\right|$ in the vortex core region and the enhanced value of $\left|\bar{\Delta}_{j}\right|$ at $j=2 n_{F}$. For comparison, the resulting $\left|\bar{\Delta}_{S C}(\mathbf{r})\right|$, calculated without $\bar{\Delta}_{j=2 n_{F}}$, is also included (red dashed line), showing no distortion of the vortex core region.

used here was first developed in Ref.[19; however, its few past applications [19, 20] have not addressed the above mentioned aspects. For the present analysis we consider a model of a $2 \mathrm{D}$ electron system under a perpendicular uniform magnetic field $\mathbf{H}=(0,0, H)$, neglecting, for the sake of simplicity, any (Zeeman or spin-orbit induced) spin splitting and assuming a singlet, $s$-wave electron pairing. The large enhancement of the cyclotron energy in the physical model systems under consideration here 11] justifies this approximation (see later).

The corresponding equations for the quasi particle states in the mean-field approximation are the BdG equations in the Landau-orbitals representation, using the magnetic Bloch basis-set wavefunctions [5, 19]:

$$
\begin{aligned}
& \sum_{n^{\prime}} \Delta_{n, n^{\prime}}(\mathbf{q}) v_{n^{\prime}}^{N}(\mathbf{q})=\left(\varepsilon^{N}(\mathbf{q})-\xi_{n}\right) u_{n}^{N}(\mathbf{q}), \\
& \sum_{n^{\prime}} \Delta_{n^{\prime}, n}^{*}(\mathbf{q}) u_{n^{\prime}}^{N}(\mathbf{q})=\left(\varepsilon^{N}(\mathbf{q})+\xi_{n}\right) v_{n}^{N}(\mathbf{q})
\end{aligned}
$$

where the single-electron (LL) energy measured relative to the chemical potential $\mu$ is given by $\xi_{n}=\hbar \omega_{c}\left(n-n_{F}\right), n=0,1,2, \ldots$, $n_{F}=\mu / \hbar \omega_{c}-1 / 2$. The matrix elements, $\Delta_{n n^{\prime}}(\mathbf{q})$, of the self-consistent pair potential, $\Delta(\mathbf{r})=$ $|V| \sum_{N} \sum_{\mathbf{q}} u_{\mathbf{q}}^{N}(\mathbf{r}) v_{\mathbf{q}}^{N *}(\mathbf{r}) \tanh \left(\varepsilon^{N}(\mathbf{q}) / 2 k_{B} T\right)$ are calculated by exploiting an expansion [21, [5],
$\Delta(\mathbf{r})=\sum_{j=0}^{\infty} \Delta_{j} \eta_{j}(\mathbf{r})$, in terms of Landau orbitals wavefunctions of a Cooper-pair (charge $2 e), \quad \eta_{j}(\mathbf{r})=\sum_{k} e^{i \pi\left(\frac{b_{x}}{a_{x}}\right) k^{2}} e^{i \frac{2 \pi k}{a_{x}} x} \varphi_{j}\left[\sqrt{2}\left(y+\frac{\pi k}{a_{x}}\right)\right]$, where $\varphi_{j}(y) \equiv\left(\sqrt{\frac{\pi}{2}} 2^{j} j !\right)^{-1 / 2} e^{-\frac{1}{2} y^{2}} H_{j}(y), \quad$ and $H_{j}(y)$ is hermite polynomial of order $j$. Here $\mathbf{a}=\left(a_{x}, 0\right), \mathbf{b}=\left(b_{x}, b_{y}\right)$ are two primitive vectors in a general rhombic vortex lattice, forming a primitive unit cell of area $a_{x} b_{y}=\pi$, corresponding to one Cooper-pair flux quantum (note that all spatial length are measured in units of the magnetic length: $\left.a_{H} \equiv \sqrt{\frac{c \hbar}{e H}}\right)$. Selfconsistency is therefore established by requiring the coefficients $\Delta_{j}$ to satisfy the equations:

$$
\begin{aligned}
\Delta_{j} & =\hbar \omega_{c}\left(\sqrt{2} \pi / a_{x}\right)\left(j ! 2^{j}\right) \lambda \sum_{n, m=n_{F}-n_{0}}^{n_{F}+n_{0}} \Gamma_{n, m}^{j} \\
& \times N_{\phi}^{-1} \sum_{\mathbf{q}} \Phi_{n m j}(\mathbf{q}) \Psi_{n, m}(\mathbf{q})
\end{aligned}
$$

where:

$$
\begin{aligned}
\Gamma_{n, m}^{j} & \equiv 2^{-n-m}(n ! m !)^{-1 / 2}(-1)^{m} \sum_{k=0}^{j}(-1)^{k} C_{k}^{m} C_{j-k}^{n}, \\
\Psi_{n, m}(\mathbf{q}) & \equiv \sum_{N} u_{n}^{N}(\mathbf{q}) v_{m}^{N *}(\mathbf{q}) \tanh \left(\varepsilon^{N}(\mathbf{q}) / 2 k_{B} T\right), \\
\Phi_{n m j}(\mathbf{q}) & =\left(\sqrt{\frac{\pi}{2}} 2^{j} j !\right)^{-1 / 2} \sum_{l} H_{n+m-j}\left[\sqrt{2}\left(q_{x}+\frac{\pi l}{a_{x}}\right)\right] \\
& \times e^{i \pi\left(\frac{b_{x}}{a_{x}}\right) l^{2}-i\left(\frac{2 \pi l}{a_{x}}\right) q_{y}-\left(q_{x}+\frac{\pi l}{a_{x}}\right)^{2}},
\end{aligned}
$$

$n_{0} \equiv\left[\omega_{D} / \omega_{c}\right], N_{\phi}$ is the number of flux lines threading the SC sample, and $\omega_{D^{-}}$the (Debye) cut-off frequency, and the matrix elements: $\Delta_{n m}(\mathbf{q})=\sum_{j=0}^{\infty} \Delta_{j, n m}(\mathbf{q})$, obeying the equations:

$$
\begin{aligned}
& \Delta_{j, n m}(\mathbf{q})=\Delta_{j}(2 \pi)^{-1 / 4}\left(j ! 2^{j}\right)^{1 / 2} \Gamma_{n, m}^{j} \sum_{l} \\
& e^{-\frac{i \pi}{4}\left(\frac{b_{x}}{a_{x}}\right) l^{2}+i \frac{2 \pi l}{a_{x}} q_{y}-\left(q_{x}+\frac{\pi l}{a_{x}}\right)^{2}} H_{n+m-j}\left[\sqrt{2}\left(q_{x}+\frac{\pi l}{a_{x}}\right)\right]
\end{aligned}
$$

Note the dimensionless coupling constant $\lambda$ in Eq2, which is related to the effective electron-electron interaction parameter $|V|$, through: $\lambda \equiv|V|\left(m^{*} / 2 \pi \hbar^{2}\right)=$ $|V| / 2 \pi a_{H}^{2} \hbar \omega_{c}$. Note also that the range of summation over the wavevectors $\mathbf{q}$ in $\mathrm{Eq} 22$ is restricted to the first magnetic Brillouin zone, which can be further reduced to a quarter, or one-sixth part of the zone, depending on whether the point symmetry of the vortex lattice is four-fold or six-fold, respectively. For the sake of simplicity, all numerical calculations have been performed here with the square-lattice geometry. The small changes associated with a different lattice geometry, e.g. the more 
common triangular geometry, are irrelevant to the purposes of the present paper.

The self-consistent solution code starts with initial values of the coefficients $\Delta_{j}$ in the expression for the matrix elements in Eq, 3 for which the calculated $4 \times\left(2 n_{0}+1\right) \times$ $\left(2 n_{0}+1\right)$ BdG matrix in the $2 \times\left(2 n_{0}+1\right)$-dimensional vector space $\left(u_{n}^{N}(\mathbf{q}), v_{m}^{N}(\mathbf{q})\right)$ is numerically diagonalized at each point $\mathbf{q}$ in the magnetic Brillouin zone. The resulting eigenvectors and eigenvalues are then used to construct the pair potential for the next iteration with the new $\Delta_{j}$ and $\Delta_{n m}(\mathbf{q})$ through Eq, 2 and 3 respectively. The iteration process continues until the values of $\Delta_{j}$ converge. Note that, due to the rotational symmetry of the vortex lattice, only $\Delta_{j}$ with $j$ integer multiple of 4 (6) for the square (triangular) lattice are different from zero. The resulting values of $\Delta_{j}$ for $j>0$, in the high magnetic fields region of well-defined Landau bands, are usually much smaller than $\Delta_{j=0}$, which corresponds to Abrikosov-lattice form of $\Delta(\mathbf{r})$, but become increasingly important upon decreasing the field below the crossover to a continuous spectrum, where the the pair-potential shows a reduced vortex core region and an oscillating behavior away from the core, which reflect the appearance of bound states. [19] Note, however, that at special small values of the LL filling factor $n_{F}+1 / 2(\propto 1 / H), \Delta_{j=2 n_{F}}$ is strongly enhanced with respect to the other coefficients with $j>0$, leading to a significant distortion of $\Delta(\mathbf{r})$ in the vortex core region, with respect to the linearly vanishing Abrikosov form (see Fig.(1)). The excess kinetic energy associated with this distortion is compensated by the extra condensation energy involved in the orbital extension over the reciprocal vortex lattice.

Self-consistent determination of the pair-potential amplitude, $\Delta_{S C}$, is also of a crucial importance in the range of relatively small $n_{F}$ (high field) values, where MQ oscillations significantly influence the transition to the SC state. Fig. (2) shows $\bar{\Delta}_{S C}\left(n_{F}\right) \equiv \Delta_{S C}\left(n_{F}\right) / \hbar \omega_{c}$ (calculated at integer values of $n_{F}$, where $\Delta_{S C}\left(n_{F}\right)$ has maxima) for the coupling constant $\lambda=0.75$ and various values of the cut-off frequency parameter $\hbar \omega_{D} / \mu$. The corresponding best fits of $\bar{\Delta}_{S C}\left(n_{F}\right)$ to a phenomenological Ginzburg-Landau (GL)-like formula:

$$
\bar{\Delta}_{G L}\left(n_{F}\right) \equiv \frac{\Delta_{G L}\left(n_{F}\right)}{\hbar \omega_{c}}=\frac{\gamma n_{F}}{n_{c 2}}\left[1-\frac{n_{c 2}}{n_{F}}\right]^{1 / 2}
$$

with two adjustable parameters, $\gamma$ and $n_{c 2}$, are also plotted in Fig.(2).

The best fitting values of $\gamma$ and $n_{c 2}$ are found to be close to $2 \gamma_{H W}$ and $2 n_{c 2}^{H W}$ respectively, where $\gamma_{H W} \approx 0.56\left(\mu / \hbar \omega_{D}\right) \sinh (1 / \lambda)$, and $n_{c 2}^{H W}=$ $\mu /\left(\hbar e H_{c 2}^{H W} / m^{*} c\right)=0.563\left(\mu / \hbar \omega_{D}\right)^{2} \sinh ^{2}(1 / \lambda)$, are the respective expressions derived within the semiclassical Helfand-Werthamer (HW) theory [18] for the order-parameter amplitude $\bar{\Delta}_{H W}\left(n_{F}\right)=\left(\gamma_{H W} / n_{c 2}^{H W}\right) n_{F}\left[1-\left(n_{c 2}^{H W} / n_{F}\right)\right]^{1 / 2}$.

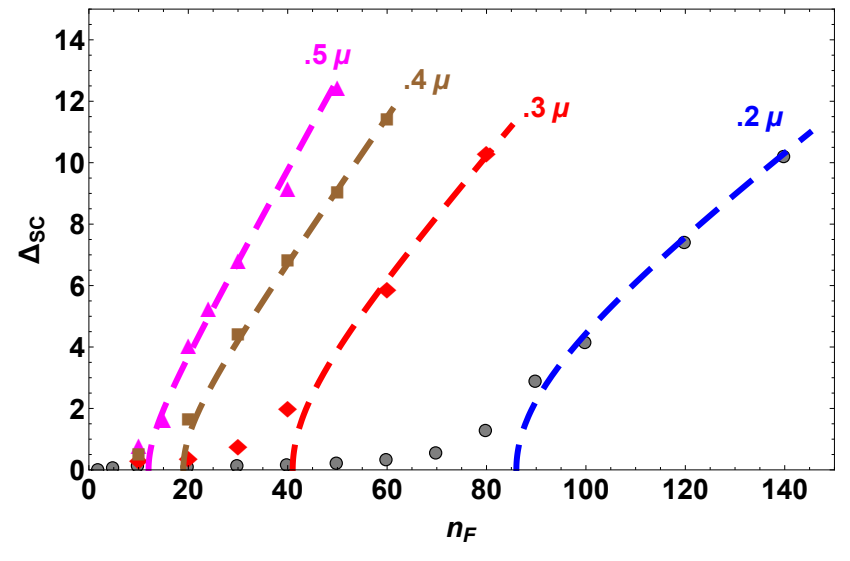

FIG. 2: Color online: Self-consistent order-parameter amplitde, $\bar{\Delta}_{S C}\left(n_{F}\right)$, (i.e. measured in units of $\left.\hbar \omega_{c}\right)$, calculated at integer values of $n_{F}$, for $\lambda=0.75$ and different values of $\omega_{D}(0.2 \mu$ - circles, $0.3 \mu$-diamonds, $0.4 \mu$-squares, and $0.5 \mu$ triangles). The corresponding best fitting curves to $\bar{\Delta}_{S C}\left(n_{F}\right)$, based on the GL-like expression are also shown (dashed lines).

Furthermore, the asymptotic $\left(n_{F} \rightarrow \infty\right)$ slope of $\bar{\Delta}_{G L}\left(n_{F}\right)$ for the best fitting parameters is found to be very close to the corresponding $\mathrm{HW}$ slope, that is: $n_{c 2} / \gamma \simeq n_{c 2}^{H W} / \gamma_{H W}=\left(\mu / \hbar \omega_{D}\right) \sinh (1 / \lambda)$ (see Fig. 3$)$. Thus, both $\Delta_{G L}\left(n_{F}\right)$ and $\Delta_{H W}\left(n_{F}\right)$ tend in the zero field limit to the well known result for the SC energy gap, $\Delta=\hbar \omega_{D} / \sinh (1 / \lambda)$. On the other hand, for $n_{F}$ values below $n_{c 2}$ (i.e. for $H>H_{c 2}=\mu m^{*} c / \hbar e n_{c 2}$ ), where $\Delta_{G L}\left(n_{F}\right)=0, \Delta_{S C}\left(n_{F}\right)$ deviates dramatically from the semiclassical theory, showing nonvanishing values, of magnitude comparable, or smaller than $\hbar \omega_{c}$. The novel quantum mixed SC state created in this field range is characterized by a cascade of normal to SC phase transitions, which crossovers to the usual SC state with a monotonically, steeply increasing order parameter amplitude for increasing $n_{F}$ (decreasing $H$ ) values around $n_{c 2}\left(H_{c 2}\right)$. This crossover closely follows the crossover of the quasi-particle spectrum from a well-defined Landau bands structure to a continuum, as can be seen in Fig.(3), where the width, $\Delta n_{F}$, of the Landau band at the chemical potential, defined by the interval of $n_{F}$ values corresponding to nontrivial self-consistent solutions, is plotted as a function of integer $n_{F}$, together with $\Delta_{S C}\left(n_{F}\right)$. It is remarkable that, in the entire fields range investigated, our calculations show that $\Delta n_{F} \simeq 0.2 \Delta_{S C}\left(n_{F}\right)$, indicating that MQ oscillations, observed in the normal state, do not suffer appreciable additional damping upon entering the quantum mixed SC state. Significant effect of the SC order parameter on the MQ oscillations can be therefore observed only in the crossover region, as found experimentally in the organic superconductor $\kappa-(E T)_{2} C u(S C N)_{2}$ [22, 23, [4]. The filling-factors range of this novel, quantum mixed SC state, estimated by $n_{c 2}-n_{c 2}^{H W} \approx n_{c 2}^{H W}=0.563\left(\mu / \hbar \omega_{D}\right)^{2} \sinh ^{2}(1 / \lambda)$, 
increases significantly (i.e. quadratically) with decreasing values of $\hbar \omega_{D} / \mu$, and much more sharply with decreasing values of $\lambda$ (see also Fig.2).

Experimental evidence for the existence of the quantum mixed SC state discussed above can be found in results reported for the high field surface superconductivity observed recently in the topological insulator $\mathrm{Sb}_{2} \mathrm{Te}_{3}$ [11. Using a simple s-wave BCS model of a 2D (circular) Fermi surface with the experimentally observed dHvA frequency, $F=36.5 \mathrm{~T}$, and cyclotron mass $m^{*}=0.065 m_{e}$, and employing the basic dimensionless constants $\lambda$ and $\hbar \omega_{D} / \mu$ of the model as adjustable parameters, we fit the zero field limit of the calculated self-consistent order parameter: $\Delta_{S C}\left(n_{F} \rightarrow \infty\right) \rightarrow \hbar \omega_{D} / \sinh (1 / \lambda)$ to the average SC energy gap derived from the STS measurements (i.e. $\simeq 13 \mathrm{meV}$ ) [11, and the semiclassical critical field ( $n_{c 2}^{H W} \approx 14$, see Fig.(3)) to the experimentally determined field of the resistivity onset downshift $H_{R}(\sim 2.5$ $\left.\mathrm{T}, n_{F} \sim 14\right) 11$. The resulting values of the adjustable parameters, $\lambda=1$ and $\hbar \omega_{D} / \mu=0.25$, imply strong coupling superconductivity with relatively small cut-off energy for the surface state of $\mathrm{Sb}_{2} \mathrm{Te}_{3}$.

Now, using the set of parameters selected above, we've calculated the self-consistent order parameter amplitude, $\Delta_{S C}\left(n_{F}\right)$, at the temperature of the experiment, $T=1.9$ $\mathrm{K}\left(k_{B} T / \mu=2.5 \times 10^{-3}\right)$, with the best fitting GL parameters; $\gamma=5.8$, and $n_{c 2}=27$, showing an extended quantum mixed state region above the crossover field $\left(n_{c 2}=27\right.$, see Fig.(3)), characterized by small Landauband width, $\Delta n_{F} \lesssim 0.1$, which seems to account for the puzzled, virtually normal state damping of the dHvA oscillation measured in this system below $H_{R}[1]$.

It should be noted that the ideal model system considered here (by ignoring effects of disorder) is aimed at emphasizing the fundamental nature of our predictions, which could be clearly observed only in sufficiently pure materials. Nevertheless, the experimental results reported in Ref.[1] indicate that for the 2D superconductivity, realized in electronic surface states with Fermi energy near a Dirac point, the stringent purity conditions are dramatically relaxed due to the drastic suppression of the cyclotron mass. Thus, with $m^{*}=0.065 m_{e}$, the LL spacing of $5 \mathrm{meV}$ at $H=3 \mathrm{~T}$, exceeds the disorder scattering relaxation rate $(\sim 3 \mathrm{meV})$, observed in $\mathrm{Sb}_{2} \mathrm{Te}_{3}$. The large scale of the cyclotron energy can also justify the neglect of the Pauli limiting effect in our model, originating in the relatively large spin-orbit splitting, estimated to be about $1 \mathrm{meV}$ in $\mathrm{Sb}_{2} \mathrm{Te}_{3}$. The latter remains smaller than $\Delta n_{F} \simeq 0.2 \Delta_{S C}\left(n_{F}\right)$ shown in Fig.3, even in the broad quantum multi-critical region.

In conclusion, we have shown here that in $2 \mathrm{D}$ electron systems with small cyclotron mass at the Fermi energy, where the effect of the magnetic field, through Landau quantization, is most pronounced, self consistency of the SC order parameter is crucial for understanding the transition to strong type-II superconductivity. In par-

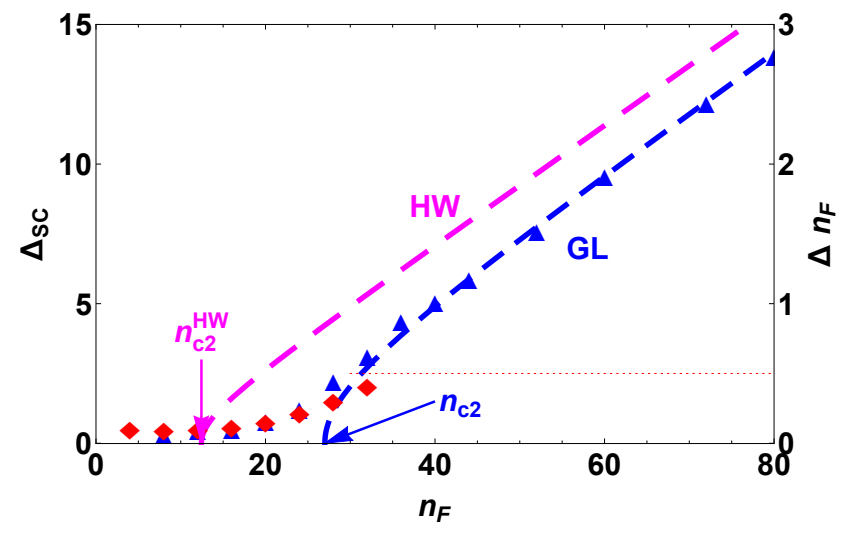

FIG. 3: Color online: Self-consistent order-parameter amplitde, $\bar{\Delta}_{S C}\left(n_{F}\right)$, calculated at integer values of $n_{F}$, for $\lambda=1.0, \hbar \omega_{D}=0.25 \mu$, and $k_{B} T=2.5 \times 10^{-3} \mu$ (blue triangles), and the best fitting curve, based on the GL-like expression (blue dashed line). Also shown is the corresponding semiclassical (HW) result ( magenta dashed line) and the self-consistent Landau bandwidth $\Delta n_{F}$ (red diamonds). The scale for $\Delta n_{F}$ on the right hand vertical axis. The horizontal red dotted segment indicates the saturation value (0.5) of $\Delta n_{F}$ (for which the bands start to overlap).

ticular, the single critical-point picture of the SC phase transition, predicted by the mean-field semiclassical theory, is smeared into a finite range multi-critical transition state, characterized by a well defined Landau-bands structure in the quasi-particle spectrum and suppressed SC order parameter amplitude. Upon decreasing magnetic field below the semiclassical $\mathrm{H}_{c 2}$ the quasi particle spectrum crossovers sharply into a continuum and the order-parameter amplitude steeply approaches the wellknown result predicted by the semi-classical (HelfandWerthamer) theory. The fields range of this quantum mixed SC phase dramatically increases upon decreasing the pairing coupling constant $\lambda$, or cutoff energy $\hbar \omega_{D}$. It is therefore expected that observable MQ oscillations can be significantly affected by the SC order parameter only in the crossover region. This seems to be the reason for the rather sporadic appearance of this effect in the diverse literature reporting on $\mathrm{dHvA}$ oscillations in the SC state. 4] 24] Our theory accounts reasonably well for the 2D SC state observed recently on the surface of the topological insulator $\mathrm{Sb}_{2} \mathrm{Te}_{3}$ 11, revealing a strong typeII superconductor with unusually low carrier density and small cyclotron effective mass, which can be realized only in the strong coupling $(\lambda \sim 1)$ superconductor limit. This unique situation is due to the proximity of the Fermi energy to a Dirac point, which implies that other materials in the emerging field of surface superconductivity, with metallic surface states and Dirac dispersion law around the Fermi energy, can show similar features.

This research was supported by E. and J. Bishop research fund at Technion. T.M. is indebted to Dingping Li and B. Rosenstein for helpful discussions. 
* Electronic address: e-mail:maniv@tx.technion.ac.il

[1] Ivan Bozovic and Charles Ahn, "A new frontier for superconductivity", nphys. 10, 893 (2014).

[2] Jian-Feng Ge et al., "Superconductivity above $100 \mathrm{~K}$ in single-layer FeSe films on doped SrTiO3", nmat. 14, 285 (2015).

[3] S. Manna et al., "Evidence for interfacial superconductivity in a bi-collinear antiferromagnetically ordered FeTe monolayer on a topological insulator", arXiv: 1606.03249 [cond-mat.supr-con].

[4] T. Maniv, V. Zhuravlev, I. D. Vagner, and P. Wyder,"Vortex states and quantum magnetic oscillations in conventional type-II superconductors", Rev. Mod. Phys. 73, 867 (2001).

[5] S. Dukan and Z. Tesanovic,"Superconductivity in a high magnetic field: Excitation spectrum and tunneling properties", Phys. Rev. B 49, 13017 (1994).

[6] S. Dukan and Z. Tesanovic," de Haas-van Alphen Oscillations in a Superconducting State at High Magnetic Fields", Phys. Rev. Lett. 74, 2311 (1995).

[7] J. Wosnitza, "Fermi surfaces of Low-Dimensional Organic Metals and Superconductors", Springer: Berlin (1996).

[8] J. Wehr, A. Niederberger, L. Sanchez-Palencia and M. Lewenstein, "Disorder versus the Mermin-WagnerHohenberg effect: From classical spin systems to ultracold atomic gases", Phys. Rev. B 74, 224448 (2006).

[9] I. Guillamon, R. Cordoba, J. Sese, J. M. De Teresa, M. R. Ibarra, S. Vieira and H. Suderow, "Enhancement of long-range correlations in a $2 \mathrm{D}$ vortex lattice by an incommensurate 1D disorder potential", Nature Phys. 10, 851 (2014).

[10] A. Maniv, V. Zhuravlev, T. Maniv, O. Ofer, R. Rommel, J. M. Müller, and J.E. Sonier, "Partially ordered vortex lattices in the high-field low-temperature mixed state of quasi two-dimensional organic superconductors", Phys. Rev. B 91, 134506 (2015).

[11] Lukas Zhao, Haiming Deng, Inna Korzhovska, Milan Begliarbekov, Zhiyi Chen, Erick Andrade, Ethan Rosenthal, Abhay Pasupathy, Vadim Oganesyan \& Lia KrusinElbaum, "Emergent surface superconductivity in the topological insulator Sb2Te3", nature communications
DOI: $10.1038 /$ ncomms9279 (2015).

[12] Haijun Zhang, Chao-Xing Liu, Xiao-Liang Qi, Xi Dai, Zhong Fang and Shou-Cheng Zhang, "Topological insulators in Bi2Se3, Bi2Te3 and Sb2Te3 with a single Dirac cone on the surface", nphys. 5 , 438 (2009).

[13] M. I. Katsnelson, "Graphen, Carbon in two Dimensions", Cambridge University Press, Cambridge (2012).

[14] Z.Tesanovic and P. Sacramento, "Landau Levels and Quasiparticle Spectrum of Extreme Type-II Superconductors", Phys. Rev. Lett. 80, 1521 (1998).

[15] V. Zhuravlev and T. Maniv, "Exact analytic GorkovGinzburg-Landau theory of type-II superconductivity in the magnetoquantum oscillations limit", Phys. Rev. B 85, 104528 (2012).

[16] T. Maniv and V. Zhuravlev, "Field-controlled conical intersections in the vortex lattice of quasi 2D pure strongly type-II superconductors at high magnetic fields". arXiv:1405.4973v1 [cond-mat.supr-con] (2014).

[17] T. Maniv, R.S. Markiewicz, I.D. Vagner and P. Wyder, "Strong Quantum Oscillations in the order parameter of 2D, type-II Superconductors", Phys. Rev. B 45, 13084 (1992). T. Maniv, A.I. Rom, I. vagner and P. Wyder, "De Haas van Alphen effect in the superconducting state of a 2D metal", Phys. Rev. B 46, 8360 (1992).

[18] E. Helfand, and N. R. Werthamer, Phys. Rev. Lett. 13, 686 (1964); Phys. Rev. 147, 288 (1966).

[19] M. R. Norman, A.H. MacDonald, and H. Akera, Phys. Rev. B 51, 5927 (1995).

[20] Kouji Yasui and T. Kita, "Theory of the de Haas-van Alphen effect in type-II superconductors". Phys. Rev. B 66, 184516 (2002).

[21] G. Eilenberger, Phys. Rev. 153, 584 (1967).

[22] P.J. van der Wel, J. Caulfield, S.M. Hayden, J. Singleton, M. Springford, P. Meeson, W. Hayes, M. Kurmoo, P. Day, Synth. Met. 70, 831 (1995).

[23] T. Sasaki, W. Biberacher, K. Neumaier, W. Hehn, K. Andres, T. Fukase,"Quantum liquid of vortices in the quasi-two-dimensional organic superconductor $\sigma$ $($ BEDT-TTF $\left.))_{2} \mathrm{Cu}(\mathrm{NCS})\right)_{2}$ ", Phys. Rev. B 5710889 (1998).

[24] T. J. B. M. Janssen, C. Haworth, S. M. Hayden, P. Meeson, M. Springford, and A. Wasserman,"Quantitative investigation of the de Haas-van Alphen effect in the superconducting state", Phys. Rev. B 57, 11698 (1998). 